\title{
Working at the interface of phylogenetics and population genetics: a biogeographical analysis of Triaenops spp. (Chiroptera: Hipposideridae)
}

\author{
A. L. RUSSELL, ${ }^{*}$ J. RANIVO,拉. P. PALKOVACS, ${ }^{*}$. M. GOODMAN $\ddagger \S$ and A. D. YODER II \\ *Department of Ecology and Evolutionary Biology, Yale University, New Haven, CT 06520, USA, +Département de Biologie \\ Animale, Université d'Antananarivo, Antananarivo, BP 106, Madagascar, $\ddagger$ Ecology Training Program, World Wildlife Fund, \\ Antananarivo, BP 906 Madagascar, §The Field Museum of Natural History, Division of Mammals, 1400 South Lake Shore Drive, \\ Chicago, IL 60605, USA, IIDepartment of Ecology and Evolutionary Biology, PO Box 90338, Duke University, Durham, \\ NC 27708, USA
}

\begin{abstract}
New applications of genetic data to questions of historical biogeography have revolutionized our understanding of how organisms have come to occupy their present distributions. Phylogenetic methods in combination with divergence time estimation can reveal biogeographical centres of origin, differentiate between hypotheses of vicariance and dispersal, and reveal the directionality of dispersal events. Despite their power, however, phylogenetic methods can sometimes yield patterns that are compatible with multiple, equally well-supported biogeographical hypotheses. In such cases, additional approaches must be integrated to differentiate among conflicting dispersal hypotheses. Here, we use a synthetic approach that draws upon the analytical strengths of coalescent and population genetic methods to augment phylogenetic analyses in order to assess the biogeographical history of Madagascar's Triaenops bats (Chiroptera: Hipposideridae). Phylogenetic analyses of mitochondrial DNA sequence data for Malagasy and east African Triaenops reveal a pattern that equally supports two competing hypotheses. While the phylogeny cannot determine whether Africa or Madagascar was the centre of origin for the species investigated, it serves as the essential backbone for the application of coalescent and population genetic methods. From the application of these methods, we conclude that a hypothesis of two independent but unidirectional dispersal events from Africa to Madagascar is best supported by the data.

Keywords: biogeography, coalescent theory, Madagascar, phylogenetics, population genetics, Triaenops

Received 14 June 2006; revision accepted 7 October 2006
\end{abstract}

Introduction

Biogeography is an inherently interdisciplinary field, drawing from the realms of ecology, evolutionary biology, systematics, geology, climatology, and geography. Over the past several decades, researchers (Emerson 2002; Raxworthy et al. 2002; Evans et al. 2003) have recognized the need to incorporate patterns of relatedness among taxa into biogeographical studies, overlaying phylogenetic trees on

Correspondence: Amy L. Russell, Arizona Research Laboratories, 1041 E. Lowell Street, BioSciences West, Room 246b, Tucson, AZ 85721, USA. Fax: (520) 626 8050; E-mail: alr2@email.arizona.edu geographical maps to determine the direction of movement within lineages and patterns of connection between areas. The resulting field of cladistic biogeography was organized around the central assumption that common branching patterns among areas of endemism for multiple codistributed species groups will reveal patterns of vicariance (Nelson \& Platnick 1981). More recently, an explicitly historical perspective has been advocated, which would attach dates to trees and directly compare the timing of cladogenic and geological events to test hypotheses of causality (Donoghue \& Moore 2003; Yoder \& Yang 2004).

While methods for dating phylogenetic trees are not without their critics (Heads 2005), dated phylogenies can 
be powerful tools for testing biogeographical hypotheses of dispersal and vicariance (Yoder et al. 2003; Near \& Keck 2005). For example, if an inferred taxon cladogram is topologically and temporally consistent with its relevant area cladogram, then a hypothesis of vicariance is inferred, whereas cladogenic events that postdate the establishment of a barrier are presumed to result from dispersal (Donoghue \& Moore 2003).

However, even the most accurately dated phylogenies may not suffice for discriminating among biogeographical hypotheses. If an observed phylogenetic topology is consistent with multiple dispersal scenarios, then a phylogenetic approach may be of limited value. Moreover, there are numerous cases in which phylogenetic resolution is weak, further limiting the power of phylogenies to reveal the biogeographical history of a species group. Poor resolution can result from conflict among gene trees, rapid bursts of speciation, or patterns of reticulation that are characteristic of recent speciation (Givnish et al. 2004; Echelle et al. 2005; Wickström et al. 2005). In such situations, a synthetic approach combining coalescent and population genetic with phylogenetic analyses can be informative for discriminating among competing biogeographical hypotheses.

In the best-case scenario, wherein a phylogeny is completely resolved and (for the sake of argument) known with certainty, an investigator can accept or reject a singleorigin hypothesis. Similarly, if the organismal group of interest is completely sampled both taxonomically and geographically, dispersal hypotheses can be identified and supported with high degrees of confidence. These are the undeniable powers of phylogenetic analysis for biogeographical study. Unfortunately, however, many biogeographers find themselves in situations in which phylogenies are poorly resolved, species radiations are incompletely sampled, and/or phylogenetic results are ambiguous with regard to directionality of geographical movements. In the latter case, coalescent and population genetic analyses offer auxiliary and complementary information for discriminating among hypotheses. This is particularly relevant for recently diverged species radiations. A distinct strength of coalescent analysis is its ability to harness information contained among alleles that reflect incomplete lineage sorting, a situation typical of young species. Coalescent-based methods have been developed to estimate ancestral population sizes, divergence times, and migration rates (Wakeley 1996; Rannala \& Yang 2003; Hey \& Nielsen 2004; Kuhner 2004). Layered upon these analyses, traditional population genetics can estimate patterns of gene flow, selection, and population growth, and thus evaluate assumptions implicit in many coalescent analyses. Therefore, it is via the synthetic application of phylogenetic, coalescent, and population genetic analyses that an investigator can derive and compare hypotheses from deep divergence events up through present population dynamics, including the various stages along this continuum. Here, we illustrate the utility of this approach with an example from Triaenops, a genus of Chiroptera distributed in eastern Africa, southwestern Asia, and Madagascar.

\section{Study organism}

Despite the potential for comparative studies among animals with varying degrees of vagility, the bats of Madagascar have largely been ignored by historical biogeographical studies. The geological history of Madagascar precludes vicariance as a biogeographical hypothesis for bats, given that the isolation of the island from Africa [165 million years ago (Ma); Storey et al. 1995] and from India (88 Ma; Coffin \& Rabinowitz 1992) considerably predates the evolution of Chiroptera (71-58 Ma; Teeling et al. 2005). Biogeographical hypotheses for Malagasy bats therefore must involve scenarios of dispersal. The capacity for flight in bats might be expected to increase the likelihood of longdistance dispersal. Chiroptera have experienced some of the longest known dispersal events among mammals, and several isolated island groups such as the Galapagos (McCracken et al. 1997), Hawaii (Stone \& Pratt 1994), and New Zealand (King 1990) have native terrestrial mammalian faunas consisting largely or exclusively of bats. Even so, long-distance flight is more likely in some chiropteran taxa than in others. Families with large wings such as Pteropodidae, or long, narrow wings (high wing-aspect ratio) such as Molossidae, are better adapted for long-distance dispersal than those with short, broad wings (low wingaspect ratio) such as Hipposideridae (Ahmad 1984).

The genus Triaenops (Chiroptera: Hipposideridae) consists of four species (Fig. 1a), of which three are endemic to Madagascar, and a fourth is distributed in eastern Africa and southwestern Asia (Peterson et al. 1995; Eger \& Mitchell 2003; Ranivo \& Goodman in press). On the basis of the modern geographical distribution of Triaenops species, we can propose various hypotheses regarding the biogeographical history of the genus (Yoder et al. 2005). Either the Malagasy Triaenops species T. rufus, T. furculus, and T. auritus form a clade that originated via a single dispersal event from Africa (Fig. 1b); or, they do not form a clade, meaning that the biogeographical history of the genus may have involved multiple independent dispersal events. Within the nonmonophyly category, the centre of origin could have been either Africa or Madagascar. If Africa was the centre of origin, one would hypothesize two independent but unidirectional dispersal events (Fig. 1c) that most probably occurred at different times. If Madagascar was the centre of origin, one would hypothesize an initial colonization of Madagascar by a proto-Triaenops ancestor, with a subsequent back dispersal to Africa (Fig. 1d). Given that these are hipposiderid bats with low wing-aspect ratios, one might assume that their proclivity for long-distance 


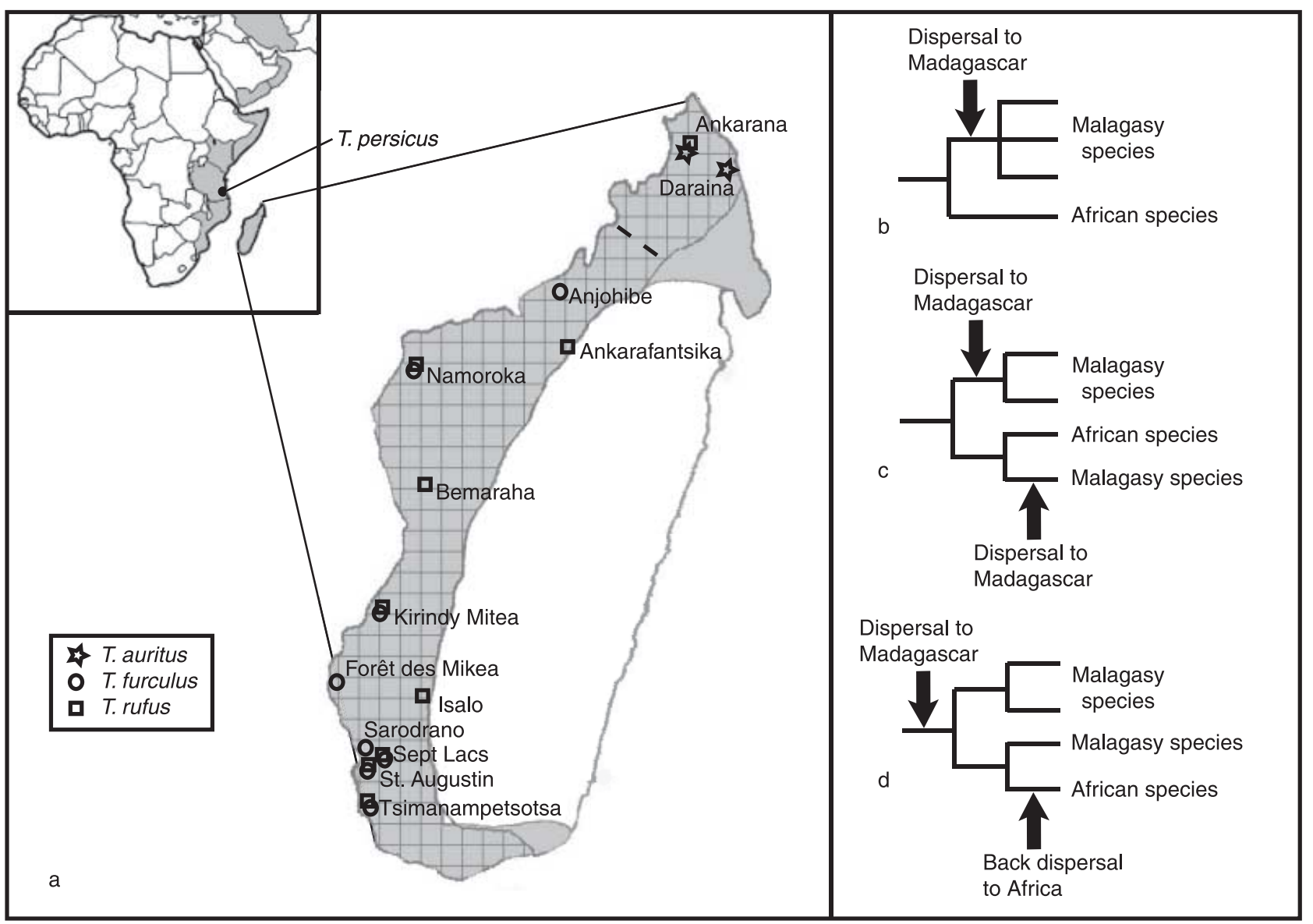

Fig. 1 (a) Geographic distributions and sampling sites of Triaenops species. The range of Triaenops persicus in mainland Africa and Asia is shown in the inset map. Ranges of Malagasy species are indicated in the blow-up of Madagascar, with the Triaenops rufus range indicated by shading and the Triaenops auritus (north of the dashed line) and T. furculus (south of the dashed line) ranges indicated by hatch marks. The dashed line is meant to indicate the approximate location of the geographical division between T. auritus and Triaenops furculus. The right panel illustrates biogeographical hypotheses proposed for Triaenops species. (b) Single dispersal hypothesis, all Malagasy species form a monophyletic clade that derives from a single dispersal event from mainland Africa. (c) Multiple dispersal hypothesis, Malagasy Triaenops are paraphyletic with respect to mainland African Triaenops. This scenario differs from that described by (d) in the direction of dispersal events.

dispersal would be diminished relative to other Malagasy bats, thereby setting a null expectation that Madagascar was colonized only once within the Triaenops lineage.

Phylogenetic analyses can be sufficient to reject the singledispersal hypothesis. If the Malagasy species are found to constitute a clade, then the hypothesis is supported (Fig. 1b). If the Malagasy lineage is shown to be polyphyletic, then additional approaches are required to differentiate among dispersal scenarios. In the case of the two most parsimonious multiple-dispersal hypotheses (Fig. 1c, d), we would expect to see significant variation in the ages of lineages resulting from different dispersal events. We use population genetic analyses to compare patterns of population substructuring and population growth among Triaenops lineages, with the expectations that (i) older lineages will have had time to develop significant genetic differentiation among geographically distinct populations, and that (ii) species resulting from more recent dispersal events may still retain the signal of population growth following their immigration and founding of a new population. We employ mitochondrial DNA (mtDNA) sequence data to compare these biogeographical hypotheses and to explore their evolutionary implications using a combination of phylogenetic, coalescent, and population genetic approaches.

\section{Methods}

\section{Sample collection}

In the context of a broad geographical survey of bats occurring in the dry habitats of the island, a considerable number of sites were visited (Table 1; Goodman et al. 2005). Animals were trapped at each site using various methods, including harp traps and mist nets placed along trails, over 


\begin{tabular}{|c|c|c|c|c|}
\hline Species & Location & $\begin{array}{l}\text { Sample } \\
\text { size }\end{array}$ & $\begin{array}{l}\text { Haplotype } \\
\text { diversity }\end{array}$ & $\begin{array}{l}\text { Nucleotide } \\
\text { diversity } \\
\text { (in } \% \text { ) }\end{array}$ \\
\hline \multirow[t]{3}{*}{ T.auritus } & RS d'Ankarana & 23 & 0.52 & 0.08 \\
\hline & Daraina & 1 & - & - \\
\hline & Total & 24 & 0.56 & 0.09 \\
\hline \multirow[t]{9}{*}{ T. furculus } & Anjohibe & 9 & 0.56 & 0.09 \\
\hline & RNI de Namoroka & 6 & 0.60 & 0.14 \\
\hline & PN de Kirindy-Mitea & 1 & - & - \\
\hline & Forêt de Mikea & 3 & 0.00 & 0.00 \\
\hline & Sarodrano & 2 & 1.00 & 0.14 \\
\hline & Sept Lacs & 4 & 0.50 & 0.14 \\
\hline & St Augustin & 7 & 0.48 & 0.07 \\
\hline & PN de Tsimanampetsotsa & 11 & 0.60 & 0.10 \\
\hline & Total & 43 & 0.70 & 0.15 \\
\hline \multirow[t]{10}{*}{ T. rufus } & RS d'Ankarana & 2 & 0.00 & 0.00 \\
\hline & PN d'Ankarafantsika & 2 & 1.00 & 1.31 \\
\hline & RNI de Namoroka & 3 & 1.00 & 1.05 \\
\hline & PN de Bemaraha & 8 & 0.96 & 0.99 \\
\hline & PN de Kirindy-Mitea & 8 & 0.89 & 0.95 \\
\hline & PN de l'Isalo & 4 & 1.00 & 0.93 \\
\hline & Sept Lacs & 2 & 1.00 & 1.29 \\
\hline & St Augustin & 9 & 0.94 & 0.63 \\
\hline & PN de Tsimanampetsotsa & 16 & 0.98 & 0.87 \\
\hline & Total & 54 & 0.97 & 0.87 \\
\hline T. persicus & Pugu Hills, Tanzania & 12 & 1.00 & 0.67 \\
\hline
\end{tabular}

Table 1 Sample size and diversity measures for each Triaenops species, given by site and as totals. We consider the two northern sites from the Triaenops furculus range to represent a distinct species, Triaenops auritus (see text for details). Total diversity measures are calculated by considering the entire species as a single gene pool. water, and at cave entrances. Voucher specimens were collected, and pectoral muscle samples were saved in a tissue storage buffer containing NaCl-saturated 25\% DMSO and $250 \mathrm{~mm}$ EDTA within $30 \mathrm{~min}$ after an animal was sacrificed. These specimens and associated tissue samples are housed in the Field Museum of Natural History, Chicago, and the Département de Biologie Animale, Université d'Antananarivo, Antananarivo.

Specimens of Triaenops rufus were collected at sites distributed relatively evenly throughout the range of the species (Fig. 1a), while specimens of Triaenops furculus were collected from sites that were more regionally clustered. We refer to these clustered regions of sampling sites as the central (Anjohibe and Namoroka) and southern regions (Kirindy Mitea, Forêt de Mikea, Sarodrano, Sept Lacs, St Augustin, and Tsimanampetsotsa; see Fig. 1a). Triaenops auritus is represented by the northernmost sampling sites (Ankarana and Daraina) in Fig. 1(a). Triaenops persicus samples were collected in the Pugu Hills, a cave site near Dar es Salaam, Tanzania.

\section{DNA isolation, amplification, and sequencing}

Total genomic DNA was isolated using a DNeasy DNA isolation kit (QIAGEN), and stored in the provided elution buffer. We used primers L14724: 5'-CGAAGCTTGATA TGAAAAACCATCGTTG-3' and H15506: 5'-AGTGGRT
TRGCTGGTGTRTARTTTC-3' (Kocher et al. 1989) to amplify approximately $750 \mathrm{bp}$ of the mitochondrial cytochrome $b$ gene. Polymerase chain reactions (PCRs) were performed in $50 \mu \mathrm{L}$ reaction volumes, each containing $2.25 \mathrm{mM} \mathrm{MgCl}_{2}$, 0.25 mm dNTPs, 2.5 U Taq DNA polymerase, $5 \mu \mathrm{L}$ Promega 10X buffer, $5 \mu \mathrm{L}$ genomic DNA, and 20 pmol of each primer. The amplification involved an initial denaturation at $94{ }^{\circ} \mathrm{C}$ for $2 \mathrm{~min}$, followed by 40 cycles of $94{ }^{\circ} \mathrm{C}$ for $45 \mathrm{~s}$, $52{ }^{\circ} \mathrm{C}$ for $45 \mathrm{~s}$, and $72{ }^{\circ} \mathrm{C}$ for $1 \mathrm{~min}$, with a final elongation step at $72{ }^{\circ} \mathrm{C}$ for $4 \mathrm{~min}$. The target fragment was then purified using either gel band excision (Gel Excision kit, QIAGEN) or PCR purification (PCR Purification Kit, QIAGEN).

The purified DNA fragment was sequenced from both directions using the BigDye terminator cycle sequencing kit version 3.0 (Applied Biosystems) in a $20-\mu \mathrm{L}$ reaction volume containing $2 \mu \mathrm{L}$ of the BigDye solution, $1.2 \mu \mathrm{L} \mathrm{ABI}$ $5 X$ sequencing buffer, 5 pmol of primer, and 100-200 ng of purified PCR product. The sequencing reaction consisted of 25 cycles of $96^{\circ} \mathrm{C}$ for $10 \mathrm{~s}, 50{ }^{\circ} \mathrm{C}$ for $5 \mathrm{~s}$, and $60^{\circ} \mathrm{C}$ for 4 min, with temperatures changing at $1{ }^{\circ} \mathrm{C}$ per second. The sequencing reactions were cleaned of unincorporated nucleotides using genCLEAN dye terminator removal plates (Genetix), and analysed on an MJ BaseStation automated sequencer.

Each individual was sequenced four to 10 times from multiple PCR reactions to resolve any ambiguities present 
in single sequencing passes. We used SEQUENCHER version 4.2 (Gene Codes) to create consensus sequences for each individual, and aligned the sequence data by eye using MACCLADE version 4.0 (Maddison \& Maddison 2000), yielding a final data set of 732 sites. All sequences were submitted to GenBank (Accession nos DQ005772-DQ005850; see Appendix).

\section{Phylogenetic analyses}

We used both Bayesian and maximum parsimony phylogenetic analyses to determine the number of independent lineages present in the data, and to examine the relationships among those lineages. We included all Triaenops sequences in these analyses, as well as sequences (Kingston et al. 2001; Sakai et al. 2003; Wang et al. 2003) from other species in the families Hipposideridae and Rhinolophidae (following the classification of Simmons 2005) as outgroups (see Appendix).

We performed parsimony analyses with PAUP* version 4.0b10 (Swofford 1998) using heuristic searches with treebisection-reconnection (TBR) branch swapping, and setting the maximum number of trees saved to 1000. Parsimony bootstrap analyses were performed with PAUP* using heuristic searches, TBR branch swapping, and 100 replicates of the random addition search option.

We used MRBAYES version 3.0b4 (Huelsenbeck \& Ronquist 2001) to perform a Bayesian analysis with flat priors. We ran four chains of 10 million generations each, with sampling every 500 generations. The chains were heated using the temperature scaling factor $\mathrm{T}=0.2$. We specified an $\mathrm{HKY}+\Gamma$ model with four rate categories and the gamma shape parameter $\alpha=0.1231$, which was estimated using MODELTEST version 3.06 (Posada \& Crandall 1998). After examining the likelihood profile using TRACER version 1.1 (http://evolve.zoo.ox.ac.uk/software.html?id = tracer), we discarded the first 5000 trees as a burn-in and constructed a 50\% majority consensus tree from the remaining 15000 trees in PAUP* (Swofford 1998). Branch lengths on the Bayesian consensus tree were estimated by maximum likelihood in PAUP* with the tree topology fixed.

\section{Coalescent analyses}

Using the species tree topology recovered by both the parsimony and Bayesian analyses (Fig. 2), we used MCMCCOAL version 1.0a (Yang 2002; Rannala \& Yang 2003) to estimate the parameters $\theta=2 N_{\mathrm{e}} \mu$, where $\mu$ is the mutation rate in substitutions/site/year) and $\tau$, the divergence time scaled by the mutation rate $\mu$. This analysis estimates $\theta$ both for tips (current species) and nodes (hypothesized ancestral species) in the tree, as well as $\tau$ for all relevant nodes; for this data set, the analysis resulted in the estimation of 10 parameters $\left(\theta_{\mathrm{A}^{\prime}}, \theta_{\mathrm{F}}, \theta_{\mathrm{P}}, \theta_{\mathrm{R}^{\prime}} \theta_{\mathrm{AF}^{\prime}} \theta_{\mathrm{PR}^{\prime}} \theta_{\mathrm{AFPR}^{\prime}}\right.$
Table 2 Evolutionary model parameters for Triaenops species. Alpha is the shape of the gamma distribution describing the among-site rate variation.

\begin{tabular}{|c|c|c|c|c|}
\hline & T. auritus & T. furculus & T. persicus & T. rufus \\
\hline Model & HKY & HKY & HKY & $\mathrm{HKY}+\Gamma$ \\
\hline$-\ln \mathrm{L}$ & 1037.756 & 1063.038 & 1170.694 & 1412.517 \\
\hline$\pi_{\mathrm{A}}$ & 0.286 & 0.289 & 0.269 & 0.268 \\
\hline$\pi_{\mathrm{C}}$ & 0.299 & 0.293 & 0.296 & 0.289 \\
\hline$\pi_{\mathrm{G}}$ & 0.136 & 0.141 & 0.170 & 0.172 \\
\hline$\pi_{\mathrm{T}}$ & 0.280 & 0.277 & 0.265 & 0.271 \\
\hline $\mathrm{Ti} / \mathrm{Tv}$ ratio & 6.012 & 8.473 & 10.525 & 18.251 \\
\hline Alpha & - & - & - & 0.243 \\
\hline
\end{tabular}

$\tau_{\mathrm{AF}}, \tau_{\mathrm{PR}^{\prime}}$ and $\tau_{\mathrm{AFPR}}-\tau_{\mathrm{AF}}$, where $\mathrm{A}=T$. auritus, $\mathrm{F}=T$. furculus, $\mathrm{P}=T$. persicus, $\mathrm{R}=T$. rufus, and multiple subscripts refer to the most recent common ancestor of the specified species). Using the default priors $(\theta=0.001 \pm$ $0.00071 ; \tau_{\mathrm{AF}}=0.0016 \pm 0.0008 ; \tau_{\mathrm{PR}}=0.005 \pm 0.00112 ; \tau_{\mathrm{AFPR}}-$ $\tau_{\mathrm{AF}}=0.0074 \pm 0.00272$ ), we first ran several short runs (100 000 samples) to fine-tune the algorithm parameters $\varepsilon_{1}$ $-\varepsilon_{5}$ until the acceptance ratios of the proposals fell within the interval $(0.1,0.7)$. We then estimated the parameters of interest from a long run (1000 000 samples), which was then repeated with a different random seed to verify the accuracy of the results via convergence.

\section{Population genetic analyses}

We used population genetic methods to test predictions of population expansion and genetic structure within the four Triaenops species. Because a fundamental assumption of these methods is that the data are not significantly influenced by selection, we first tested for evidence of selection using the McDonald-Kreitman test (McDonald \& Kreitman 1991) on pairs of sister species as implemented in DNASP version 4.0 (Rozas et al. 2003). Given the negative results of those tests, indicating a lack of significant selective pressure, we proceeded with further population genetic analyses.

For each species, we used MODELTEST version 3.06 to determine the best-fitting model of evolution, and to estimate evolutionary parameters such as nucleotide frequencies and transition/transversion ratios (see Table 2). Using these species-specific parameter values, we employed ARLEQUIN version 2.001 (Schneider et al. 2001) to calculate descriptive measures such as haplotype diversity $(h)$ and nucleotide diversity $(\pi)$. We also examined patterns of genetic structuring among sampling sites using an analysis of molecular variance (AMOVA), pairwise $F_{\mathrm{ST}}$ measures, and Mantel tests of correlations between genetic and geographical distances (Slatkin 1991; Excoffier et al. 1992). 


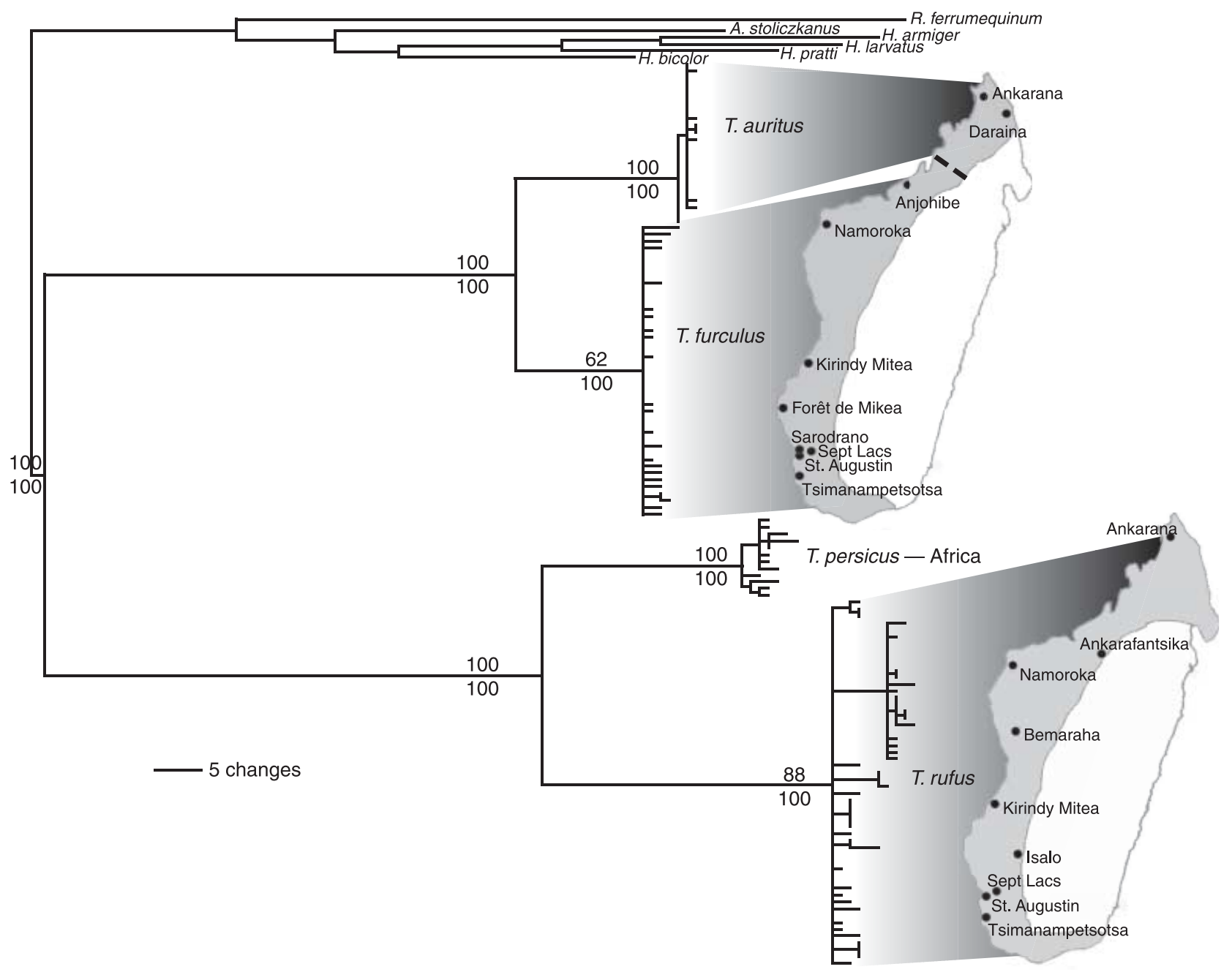

Fig. 2 Bayesian phylogeny of Triaenops species, with geographical distributions of Malagasy lineages. Support measures of species-level lineages are given as Bayesian posterior probabilities (\%) below each branch and maximum parsimony bootstrap support values above each branch. Samples of Triaenops persicus represent a single population in Tanzania.

We tested hypotheses of population expansion using comparisons of the diversity statistics $\pi$ and $h$, neutrality tests such as Fu's (1997) $F_{\mathrm{S}^{\prime}}$ Fu \& $\mathrm{Li}^{\prime} \mathrm{s}(1993) F^{*}$ and $D^{*}$, Ramos-Onsins and Rozas's (2002) $R_{2}$, and von Haeseler et al.'s (1995) expansion coefficient $S / d$, as implemented in the software package DNASP. Under a model of sudden population expansion, haplotype diversity $(h)$ is expected to be high as most new haplotypes are maintained in the population; concomitantly, because these new haplotypes are all recently derived, the nucleotide diversity $(\pi)$ of the population is predicted to be low. A similar relationship is described by von Haeseler et al.'s (1995) expansion coefficient, $S / d$, or the ratio between the number of polymorphic sites $(S)$ and the mean pairwise sequence difference $(d)$. Fu (1997) showed that comparisons of statistical neutrality tests could be used to infer the demographic history of a population. If Fu's (1997) $F_{\mathrm{S}}$ is significant but $\mathrm{Fu} \& \mathrm{Li}$ 's
(1993) $F^{*}$ and $D^{*}$ are not, then a history of population growth or range expansion is indicated; the reverse situation suggests the influence of background selection. Ramos-Onsins \& Rozas (2002) recently described another powerful statistic for detecting population growth, $R_{2}$.

As a more rigorous analysis of population growth, we used LAMARC version 2.0.2 (Kuhner 2006) to estimate the population growth parameter $g$ for each of the three Malagasy species. For each species, we ran three independent runs of a Bayesian search. Each run consisted of three replicates of one short and one long chain. The short chains were 10000 steps long, with 500 trees sampled every 20 steps and a burn-in of 1000 trees. The long chains were 1000000 steps long, with 10000 trees sampled every 100 steps and a burn-in of 1000 trees. Priors for the growth rate were set at the maximum allowable range of $-500-$ 15000. 


\begin{tabular}{llll}
\hline & Prior & Posterior $\pm \mathrm{SD}$ & Parameter estimate $\pm \mathrm{SD}$ \\
\hline$\theta_{\mathrm{A}}$ & $0.001 \pm 0.00071$ & $0.00202 \pm 0.00081$ & $N_{\mathrm{e}}=5050 \pm 2025$ \\
$\theta_{\mathrm{F}}$ & $0.001 \pm 0.00071$ & $0.00278 \pm 0.00096$ & $N_{\mathrm{e}}=6950 \pm 2400$ \\
$\theta_{\mathrm{P}}$ & $0.001 \pm 0.00071$ & $0.00555 \pm 0.00131$ & $N_{\mathrm{e}}=13875 \pm 3275$ \\
$\theta_{\mathrm{R}}$ & $0.001 \pm 0.00071$ & $0.01257 \pm 0.002$ & $N_{\mathrm{e}}=31425 \pm 5000$ \\
$\theta_{\mathrm{AF}}$ & $0.001 \pm 0.00071$ & $0.00284 \pm 0.001$ & $N_{\mathrm{e}}=7100 \pm 2500$ \\
$\theta_{\mathrm{PR}}$ & $0.001 \pm 0.00071$ & $0.00180 \pm 0.001$ & $N_{\mathrm{e}}=4500 \pm 2500$ \\
$\theta_{\mathrm{AFPR}}$ & $0.001 \pm 0.00071$ & $0.01293 \pm 0.00198$ & $N_{\mathrm{e}}=32325 \pm 4950$ \\
$\tau_{\mathrm{AF}}$ & $0.0016 \pm 0.0008$ & $0.00229 \pm 0.00086$ & $57250 \pm 21500 \mathrm{BP}$ \\
$\tau_{\mathrm{PR}}$ & $0.005 \pm 0.00112$ & $0.00556 \pm 0.00116$ & $139000 \pm 29000 \mathrm{BP}$ \\
$\tau_{\mathrm{AFPR}}-\tau_{\mathrm{AF}}$ & $0.0074 \pm 0.00272$ & $0.00509 \pm 0.00209$ & $127250 \pm 52250 \mathrm{BP}$ \\
$\tau_{\mathrm{AFPR}}$ & - & 0.00738 & $184500 \mathrm{BP}$ \\
\hline
\end{tabular}

Table 3 Estimates of population size parameters $\left(\theta_{i}\right)$ and ages of nodes $\left(\tau_{i}\right)$ in the Triaenops species tree, scaled by mutation rate. Estimates of $\theta$ are given for each species and for ancestral species indicated by the nodes in the species tree. Parameter estimates $\left(N_{\mathrm{e}^{\prime}}\right.$ absolute ages of nodes) were derived assuming $\mu=4 \times 10^{-8}$ substitutions/site/year (Hulva et al. 2004) and a generation time of 5 years.

\section{Results}

\section{Phylogenetic analyses}

Both parsimony and Bayesian phylogenetic analyses reveal a phylogeny that is consistent with four reciprocally monophyletic species lineages. Because the Malagasy endemic Triaenops rufus is supported as a lineage separate from, but sister to, the mainland African species Triaenops persicus, a single origin hypothesis is not supported (Fig. 2). Furthermore, a separate species lineage, Triaenops auritus, is supported in northern Madagascar and is genetically and geographically distinct from, but sister to, Triaenops furculus. Measures of HKY-corrected genetic diversity further illustrate the differentiation of the previously subsumed $T$. auritus species. Intraspecific diversity measures range from $0.28 \%$ for T. auritus to $2.19 \%$ for T. rufus, while diversity measures between sister-species pairs are $4.57 \%$ for $T$. auritus-T. furculus and $8.19 \%$ for T. rufus-T. persicus. These measures correspond well with those typically observed at cytochrome $b$ between sister species within Chiroptera $($ mean $=6.83 \%$, range $=2.50-16.42 \%$; Bradley \& Baker 2001 $)$.

\section{Coalescent analyses}

We used a coalescent-based analysis (Rannala \& Yang 2003) to estimate the divergence time parameter $\tau$, as well as the population diversity parameter $\theta$, for each node (and tip, in the case of $\theta$ in our Triaenops species tree (Table 3). Using a mutation rate from the cytochrome $b$ gene of Pipistrellus bats (Hulva et al. 2004) and assuming a generation time of 5 years (S.M.G., personal observation), we calculated $N_{\mathrm{e}}$ and the absolute ages of nodes from the mutation rate-scaled parameters $\theta$ and $\tau$, respectively. The species tree was constrained to the observed topology [(T. auritus, T. furculus), (T. persicus, T. rufus)] for these analyses.

Estimates of the population diversity parameter $\theta$ indicate significant differences in the sizes of current and ancestral species. The clade containing T. auritus and T. furculus is distinguished by small population sizes for both current $\left(\theta_{\mathrm{A}}=0.00202 \pm 0.00081 ; \theta_{\mathrm{F}}=0.00278 \pm 0.00096\right)$ and ancestral populations $\left(\theta_{\mathrm{AF}}=0.00284 \pm 0.001\right)$. Conversely, while the current population sizes for T. persicus and T. rufus are much larger $\left(\theta_{P}=0.00555 \pm 0.00131 ; \theta_{R}=0.01257 \pm 0.002\right)$, our analyses indicate that this species pair evolved from a much smaller ancestral population $\left(\theta_{\mathrm{PR}}=0.00180 \pm 0.001\right)$. The earliest inferred species in the Triaenops lineage, represented by the root node, is estimated to have had a larger effective population size, similar to that of modern T. rufus $\left(\theta_{\mathrm{AFPR}}=0.01293 \pm 0.00198\right)$.

Divergence age estimates indicate that the sister species T. auritus and T. furculus diverged much more recently $\left(\tau_{\mathrm{AF}}=0.00229 \pm 0.00086\right)$ than the sister species $T$. rufus and T. persicus $\left(\tau_{\mathrm{PR}}=0.00556 \pm 0.00116\right)$ (Fig. 3$)$. The root node for Triaenops is estimated as being not much older than the divergence of $T$. rufus and T. persicus $\left(\tau_{\mathrm{AFPR}}=0.00738\right)$. These analyses place the root node of Triaenops at 184500 вр. The divergence event separating T. rufus and T.persicus dates to no earlier than $139000 \pm 29000 \mathrm{BP}$, and the divergence of T. auritus and T. furculus dates to $57250 \pm 21500 \mathrm{BP}$.

\section{Population genetic analyses}

Hierarchical analyses of molecular variance (AMOva) and pairwise $F_{\mathrm{ST}}$ tests reveal different patterns of genetic structuring between $T$. furculus and T. rufus. T. furculus is characterized by significant genetic structuring between the central (Anjohibe and Namoroka) and southern (Kirindy Mitea, Forêt de Mikea, Sarodrano, Sept Lacs, St Augustin, and Tsimanampetsotsa) geographical regions (see Fig. 1a), but high levels of genetic mixing within those regions. An AMOVA shows significant structure between the two regional groups $\left(\Phi_{\mathrm{CT}}=0.608, P=0.038\right)$, though populations within regional groups are thoroughly mixed (central region: $\Phi_{\mathrm{ST}}=0.049, P=0.253$; southern region: $\Phi_{\mathrm{ST}}=-0.115, P=$ 0.818 ). A remarkably different pattern is observed in $T$. rufus. 


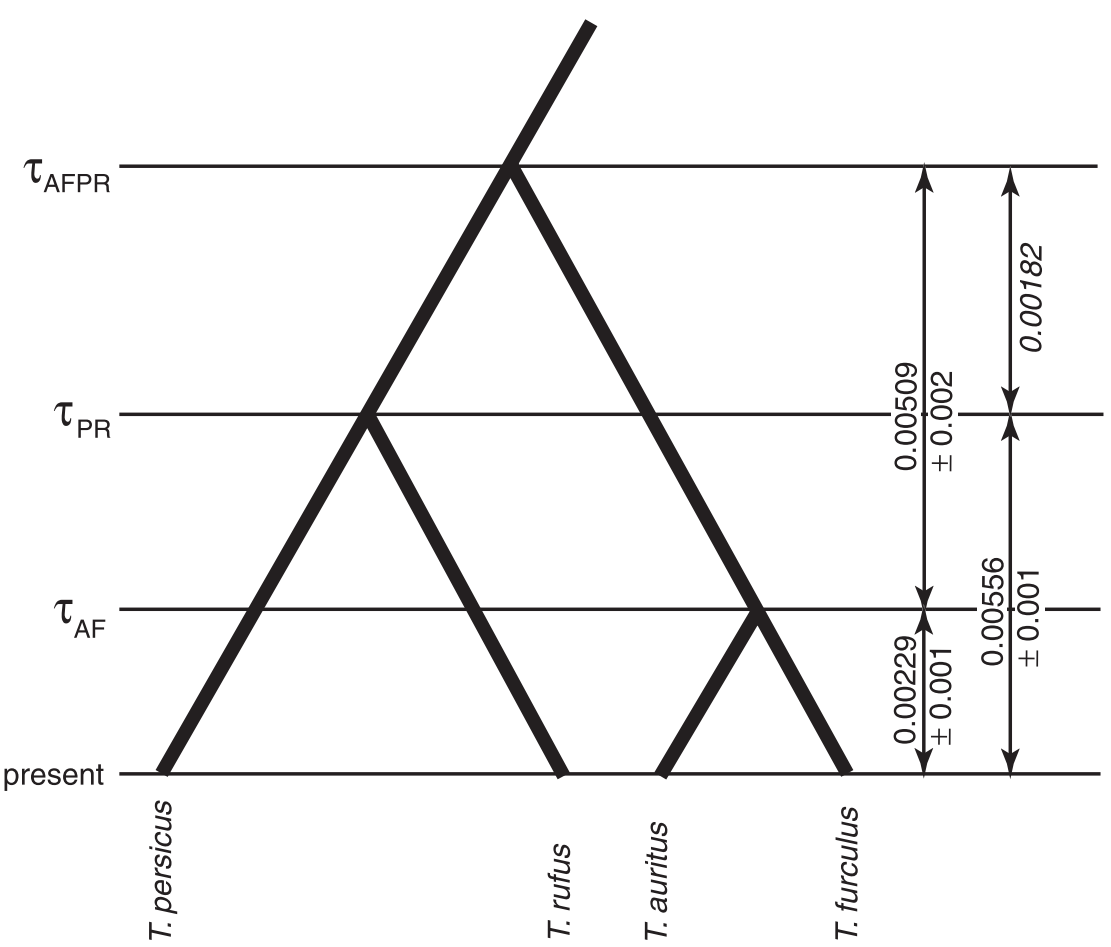

Fig. 3 Mutation rate-scaled divergence times for nodes in the Triaenops species tree. Internode intervals inferred from direct estimates (e.g. $\tau_{\mathrm{AFPR}}-\tau_{\mathrm{PR}}$ ) are given in italics.

Table 4 Results of demographic analyses of Malagasy Triaenops species, including the expected results under a scenario of population expansion. Excepting coalescent estimates of population growth, results for Triaenops furculus are presented separately for the structured central and southern regions.

\begin{tabular}{|c|c|c|c|c|c|}
\hline & T.auritus & $\begin{array}{l}\text { T. furculus } \\
\text { (central) }\end{array}$ & $\begin{array}{l}\text { T. furculus } \\
\text { (south) }\end{array}$ & T.rufus & Expansion \\
\hline Haplotype diversity $(h)$ & 0.590 & 0.562 & 0.468 & $0.966+$ & High \\
\hline Nucleotide diversity $(\pi), \%$ & 0.091 & $0.139^{* *}$ & 0.083 & $0.872^{* *}$ & Low \\
\hline Expansion coefficient $(S / d)$ & 7.463 & 6.565 & 5.848 & 8.144 & High \\
\hline Fu \& Li's (1993) F* & -0.563 & -2.181 & -0.464 & -2.294 & Not significant \\
\hline $\mathrm{Fu} \& \mathrm{Li}^{\prime} \mathrm{s}(1993) D^{*}$ & -0.189 & -1.956 & -0.240 & -2.408 & Not significant \\
\hline Fu's (1997) $F_{S}$ & -1.895 & -1.754 & -1.208 & $-19.268^{* *}$ & Significant \\
\hline Ramos-Onsins \& Rozas's (2002) $R_{2}$ & 0.085 & 0.133 & 0.096 & $0.054^{*}$ & Significant \\
\hline Population growth $(g)$ & 13301.39 & 4715.58 & & 949.31 & \\
\hline $95 \% \mathrm{CI}$ of $g$ parameter & $(905.41,14971.63)$ & $(269.35,14726.08)$ & & $(467.45,2024.40)$ & \\
\hline
\end{tabular}

${ }^{*} P<0.05 ; * P<0.01 ;$ tobserved value $>$ upper $95 \% \mathrm{CI}$.

Analyses of population structuring reveal no significantly distinct gene pools within T. rufus, indicating instead that the species represents a single deme over its entire range. We found no evidence of genetic structuring among populations in an AMOVA $\left(\Phi_{\mathrm{ST}}=-0.028, P=0.725\right)$, and all pairwise $F_{\mathrm{ST}}$ estimates were insignificant $\left(F_{\mathrm{ST}}=-0.496-\right.$ 0.192). Over a geographical distribution that spans the entire $1200 \mathrm{~km}$ length of the island, we found no evidence of isolation by distance $(r=0.212, P=0.133)$. To test whether the structure present among $T$. furculus sites reflects a real segregation of gene pools and not just a sampling artefact, we performed an AMovA among T. rufus sites selected to mirror the gaps in our sampling of T. furculus (Ankarafantsika and Namoroka vs. Kirindy Mitea, Isalo, Sept Lacs, St Augustin, and Tsimanampetsotsa). This analysis further illustrates the real lack of genetic structure within T. rufus $\left(\Phi_{\mathrm{ST}}=-0.07 \% ; P=0.334\right)$ as compared with $T$. furculus.

We used diversity statistics, neutrality tests, and coalescent estimates of the population growth rate $g$ to look for signals of population growth in each of the three Malagasy Triaenops species (Table 4; Fig. 4). Analyses of T. auritus strongly indicate a demographic history of a small but constant population size, with insignificant results for all statistical tests and a nearly flat posterior probability distribution for $g$. Demographic analyses of the structured central and southern populations of T. furculus also support 


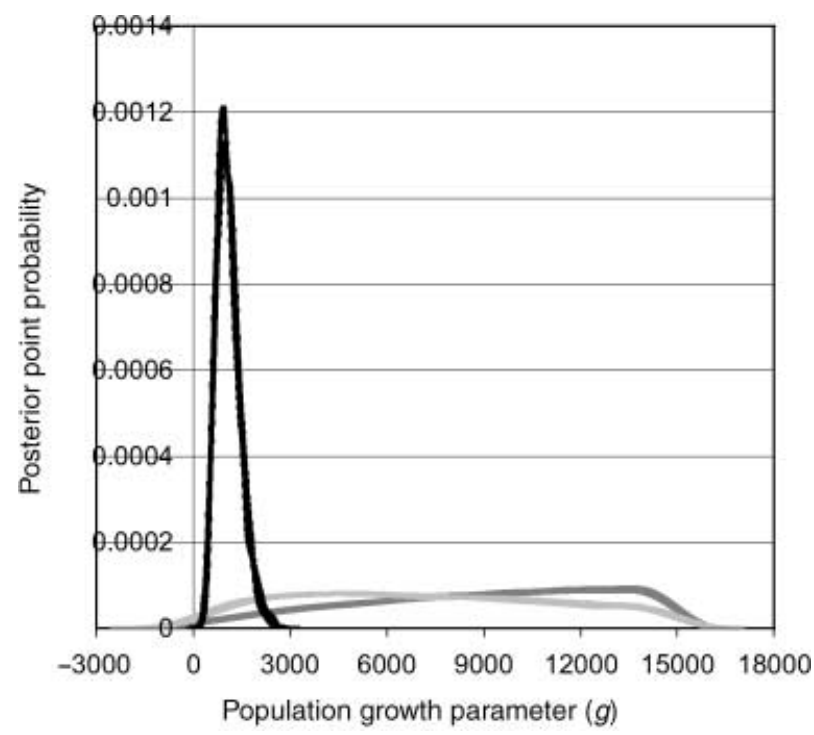

Fig. 4 Posterior probability distributions of population growth parameter $(g)$ for Malagasy species. Each species is represented by three independent runs. Triaenops rufus is shown in black, Triaenops auritus in dark grey, and Triaenops furculus in light grey.

a model of a small but constant population size. The nucleotide diversity for the central population of this species is significantly low, but the haplotype diversity is well within the $95 \%$ CI (upper 95\% confidence limit $=0.886$ ). Notably, the two tests shown to be the most powerful at detecting population growth, Fu's (1997) $F_{\mathrm{S}}$ and Ramos-Onsins \& Rozas's (2002) $R_{2}$, are not significant. Correspondingly, estimates of $g$ have a nearly flat posterior probability distribution. In contrast, a history of population growth or range expansion is strongly indicated for $T$. rufus. This species is characterized by very high haplotype and significantly low nucleotide diversities, and the neutrality tests $F_{\mathrm{S}}$ and $R_{2}$ are significant, but $F^{*}$ and $D^{*}$ are not. Furthermore, estimates of $g$ reveal a strong signal of population growth in this species with $g=949.31(\mathrm{CI}=467.45,2024.40)$, averaged across three runs.

\section{Discussion}

\section{Historical biogeography of Triaenops}

Parsimony and Bayesian phylogenetic analyses reveal a phylogeny that is consistent with four reciprocally monophyletic species lineages (Fig. 2). Because the Malagasy endemic Triaenops rufus is supported as a lineage separate from, but sister to, the mainland African species Triaenops persicus, a single origin hypothesis is not supported. Previous work had suggested that T. rufus and T. persicus be synonymized (Hill 1982; Garbutt 1999), but the long branches separating the two clades, reflecting considerable genetic differentiation between them, indicates that the proposed synonymy be rejected. Furthermore, our data support Ranivo \& Goodman's (in press) morphological analyses in recognizing a species lineage in northern Madagascar, Triaenops auritus, which is genetically and geographically distinct from, but sister to, Triaenops furculus.

Using the Triaenops phylogeny, we can address the competing biogeographical hypotheses concerning the origin of the Malagasy species T. auritus, T. furculus, and T. rufus. Our data clearly reject a single-dispersal hypothesis (Fig. 1b), which predicts that lineages endemic to Madagascar form an exclusive clade. Instead, we find that Malagasy Triaenops are paraphyletic with respect to the mainland African species T. persicus, indicating that the distribution of Malagasy and African Triaenops involved at least two independent dispersal events (Fig. 1c, d). The phylogenetic results in isolation, however, cannot detect the direction of dispersal events, and are therefore unable to discriminate between the dispersal hypotheses depicted in Fig. 1(c, d). In other words, the phylogeny does not allow us to determine whether Madagascar or Africa was the geographical centre of origin for this species radiation.

\section{Timing of dispersal events}

The multiple-dispersal models suggested by our phylogenetic analyses suggest an analysis of the timing and direction of these events. A population genetic model incorporating parameters of population substructuring might posit that, given rough equalities in geographical range and dispersal ability, older lineages will tend to be more geographically structured than younger lineages (Sawyer 1977; Castric \& Bernatchez 2003). Under the unidirectional dispersal hypothesis, we would then expect to detect significant differences between the extent of population substructuring in the Malagasy lineages, with the extent of substructuring positively correlated with the age of the lineage. Under the bidirectional hypothesis, we expect to find equivalent patterns of substructure within the two Malagasy lineages, while the mainland species T. persicus would lack significant population substructure.

We used a coalescent-based analysis (Rannala \& Yang 2003 ) to estimate the divergence time parameter $\tau$, as well as the population diversity parameter $\theta$, for each node (and tip, in the case of $\theta$ ) in our Triaenops species tree (Table 3 ). Divergence age estimates indicate that the sister species T. auritus and T. furculus diverged much more recently than the sister species T. rufus and T. persicus (Fig. 3). The root node for Triaenops is not much older than the divergence of $T$. rufus and T. persicus. These divergence age estimates are very similar to the prior settings, possibly indicating a lack of signal in the data for estimating time parameters; therefore, we advocate extreme caution in interpreting these results. Additional error may result from bias in the 
estimation method. Using data from great apes, Rannala \& Yang (2003) found that their analyses consistently underestimated divergence dates. To alleviate this problem, the authors used a highly informative prior based on fossil information for the chimp-human node, which resulted in more reasonable estimates of $\tau$ throughout the tree. Unfortunately, such fossil evidence is lacking in African hipposiderid bats, and the absolute dates presented here must be taken as preliminary estimates to be verified with additional data.

\section{Implications from population genetic analyses}

Although we are not able to date nodes in the species tree with a great deal of confidence, our population genetic model does predict significant differences in the extent of population structuring within species. Specifically, if the unidirectional dispersal hypothesis is correct and if intraisland dispersal capacities are roughly equivalent between species, we expect to detect significant differences in patterns of population substructuring between the two largely sympatric Malagasy lineages, T. rufus and T. furculus, with the extent of substructuring positively correlated with the age of the lineage. Alternatively, if the bidirectional hypothesis is correct, we expect to find equivalent patterns of substructure within the two more geographically widespread Malagasy species, T. rufus and T. furculus, while the mainland species $T$. persicus would lack significant population substructure. Unfortunately, our sampling of T. persicus comes from only a single location; therefore, tests of this model will be limited to comparisons of Malagasy species.

Hierarchical AMOvAs and pairwise $F_{\mathrm{ST}}$ tests reveal markedly different patterns of genetic structuring between T. furculus and T. rufus. T. furculus is characterized by significant genetic structuring between the central and southern geographical regions (see Fig. 1a), but high levels of genetic mixing within those regions. A notably different pattern is observed in T. rufus. Analyses of population structuring, including AMOvAs, pairwise $F_{\mathrm{ST}} \mathrm{s}$, and Mantel tests, reveal no significantly distinct gene pools within $T$. rufus, indicating instead that the species represents a single deme over its entire range. Under a population genetic model incorporating population substructure and assuming that differences in population structure do not reflect any current differences in intra-island dispersal behaviour, these results support the unidirectional dispersal hypothesis, and furthermore suggest that the dispersal event in the $T$. auritus $/ T$. furculus lineage predated that in the T. rufus lineage.

While it seems clear that multiple dispersal events between Madagascar and mainland Africa have occurred in the history of Triaenops, we expect that these events have been rare and that an evolutionarily successful dispersal would involve only one or a few individuals. Furthermore, theory predicts that significant population growth should follow the successful immigration of a small founding population (Carson \& Templeton 1984); however, the genetic signal of that demographic growth will dissipate over time. If we are correct that the dispersal event in the T. auritus/T. furculus lineage predated that in the T. rufus lineage, then we expect to detect a signal of population growth following immigration more clearly in T. rufus than in T. furculus or T. auritus. We used diversity statistics, neutrality tests, and coalescent estimates of the population growth parameter $g$ to test this prediction (Table 4; Fig. 4). Analyses of both T. auritus and the structured central and southern populations of T. furculus strongly indicate a demographic history of a small but constant population size, with insignificant results for nearly all statistical tests. Furthermore, while maximum posterior estimates of $g$ are positive, the posterior probability distributions for both species are nearly flat and do not yield a clear signal of growth. In contrast, both coalescent and summary statistical analyses strongly indicate a history of population growth or range expansion in T. rufus. While it is possible that these analyses reflect a population bottleneck in Madagascar that is not associated with the colonization process, we consider that an unlikely scenario, as such an event probably would have had similar demographic effects on T. auritus and $T$. furculus. In summary, these analyses appear to support the unidirectional dispersal hypothesis, with T. rufus having undergone population expansion following a relatively recent dispersal event and the T. auritus/T. furculus lineage deriving from an older dispersal event with subsequent speciation and intraspecific population structuring.

While relatively simplistic, the population genetic model we consider here illustrates how the conclusions drawn from genetic analyses depend to a large extent on the complexity of the model being used. A model incorporating population structure appears to support the unidirectional dispersal hypothesis, with T. rufus representing the earlier dispersal event. The topology of the phylogeny appears to support the conclusions of this model, with T. rufus being the most closely related Malagasy species to the presumed source species, T. persicus. However, the range of T. persicus covers a much larger geographical area than is represented in our sample, from Mozambique north to Iran and southwestern Pakistan, and is categorized into two subspecies (Hill 1982). A more thorough sampling of T. persicus might indicate that the T. rufus and T. auritus/T. furculus lineages differ substantially in their areas of origin, or that Tanzanian T. persicus do indeed represent a back migration to mainland Africa while other populations of T. persicus have served as populations of origin for Malagasy Triaenops. Indeed, the signature of population growth in our sample of T. persicus makes the latter scenario an intriguing possibility (results not shown). 


\section{Conclusions}

We have shown how coalescent and population genetic approaches may potentially discriminate among competing biogeographical hypotheses of dispersal posed by phylogenetic analyses. In general, we advocate initial analyses in which a rigorously dated phylogeny, preferably incorporating information from fossil samples, is compared with a corresponding area cladogram to distinguish between hypotheses of dispersal and vicariance. In cases wherein the data reject vicariance, and if the topology of the phylogeny is consistent with more than one hypothesis of dispersal, coalescent and population genetic methods can then be used to test expectations regarding measures of genetic diversity, population structuring, and population growth in light of the relative timing of dispersal events. We emphasize the importance of using population genetic analyses to test assumptions implicit in hypotheses comparing levels of genetic diversity. Population genetic forces may increase diversity in a species, as in the case of balancing selection or population structuring, or they may decrease diversity, as in the case of directional selection or a population bottleneck. Furthermore, forces such as selection and recombination will tend to affect diversity at a specific locus, while variation in population size or patterns of structure will affect diversity throughout the genome equally. Therefore, specific hypotheses at any level within this methodological framework will be strongly dependent on the system being examined.

We have used this synthesis of phylogenetic, coalescent, and population genetic approaches to address the historical biogeography of trident bats (Triaenops spp.). These analyses clearly reject a single origin hypothesis, and indicate a history of multiple dispersal events in this species group. The most parsimonious hypotheses involving multiple dispersals include either two dispersals from Africa to Madagascar or an early dispersal to Madagascar followed by a back dispersal to Africa. Coalescent-based estimates of divergence times are not conclusive for distinguishing between these hypotheses, but observed population genetic patterns, including relative measures of genetic diversity, patterns of genetic structuring, and patterns of population growth, suggest the highest support for two diachronic dispersal events from Africa to Madagascar, given the assumptions of a relatively simplistic population genetic model. We prefer this hypothesis for the reasons outlined here; however, there are a number of alternative models that are consistent with our observed results, including biogeographical models involving more than two dispersal events.

The naivety of the population genetic model considered here points to a need for quantitative phylogeographical models that explicitly incorporate geography, climatology, dispersal, and coalescent-based population genetics in a unified analytical framework. For example, for most of the year, prevailing winds in the Mozambique Channel flow from east to west, towards mainland Africa (Hastenrath \& Greischar 1991). A unified analysis of the biogeography of Triaenops might attach a higher likelihood to the bidirectional rather than the unidirectional dispersal hypothesis, given this fact. We anticipate that future work will increasingly utilize an approach testing predictions at multiple biological levels to reach informed conclusions regarding evolutionary and biogeographical history. We hope that the abundance of multidisciplinary data used to answer such questions will stimulate the development of much-needed analytical methods and models.

Further exploration of the biogeography of Triaenops should focus on better sampling of $T$. persicus to give a broader perspective on the diversity and historical demography of mainland African populations. Additionally, the inclusion of multiple unlinked loci is necessary to verify patterns observed at the cytochrome $b$ locus and to yield more rigorous estimates of species divergence dates. Our study highlights ecological questions that remain to be answered for Malagasy Triaenops; namely, whether $T$. auritus and $T$. furculus differ significantly in their diet and echolocation characteristics, how Malagasy Triaenops disperse across the landscape, and whether differences in genetic structuring can be explained by differences in patterns of regional dispersal.

\section{Acknowledgements}

This manuscript greatly benefited from comments from Adalgisa Caccone, Scott Cardiff, Cassandra Miller-Butterworth, Jeffrey Powell, Ziheng Yang, several anonymous reviewers, and the Caccone/ Powell laboratories, as well as from attendees and organizers of the Phylogeny and Phylogeography Workshop at the Mathematical Biosciences Institute of The Ohio State University. We are grateful to the Direction des Eaux et Forêts and Association National pour la Gestion des Aires Protégées for issuing permits to conduct faunal surveys. The fieldwork associated with this study was undertaken with the financial aid of the Ellen T. Smith and Marshall Field funds of the Field Museum of Natural History, John D. and Catherine T. MacArthur Foundation, National Geographic Society (6637-99 and 7402-03), and Volkswagen Foundation and under the protocol de collaboration between the Universite d'Antananarivo, WWF-Madagascar, and the Field Museum of Natural History. Laboratory work associated with this study was supported by financial assistance from the Bay and Paul Foundations and an NSF Career Award to A.D.Y. A.L.R. was supported by a Gaylord Donnelley Postdoctoral Fellowship from the Yale Institute for Biospheric Studies for the duration of the project. Specimens from the Sept Lacs region were made available by Frontier-Madagascar (Society for Environmental Exploration) through Annette Olsson. We thank William Stanley (Field Museum of Natural History) for providing Triaenops persicus samples collected in Tanzania. 


\section{References}

Ahmad A (1984) A comparative study on flight surface and aerodynamic parameters of insects, birds and bats. Indian Journal of Experimental Biology, 22, 270-278.

Bradley RD, Baker RJ (2001) A test of the genetic species concept: cytochrome- $b$ sequences and mammals. Journal of Mammalogy, 82, 960-973.

Carson HL, Templeton AR (1984) Genetic revolutions in relation to speciation phenomena: the founding of new populations. Annual Review of Ecology and Systematics, 15, 97-131.

Castric V, Bernatchez L (2003) The rise and fall of isolation by distance in the anadromous brook charr (Salvelinus fontinalis Mitchill). Genetics, 163, 983-996.

Coffin MF, Rabinowitz PD (1992) The Mesozoic East African and Madagascan continental margins: stratigraphy and tectonics. In: Geology and Geophysics of Continental Margins (eds Watkins JS, Feng Z, McMillen KJ), pp. 207-246. American Association of Petroleum Geologists, Tulsa, Oklahoma.

Donoghue MJ, Moore BR (2003) Toward an integrative historical biogeography. Integrative and Comparative Biology, 43, 261-270.

Echelle AA, Carson EW, Echelle AF et al. (2005) Historical biogeography of the New World pupfish genus Cyprinodon (Teleostei Cyprinodontidae). Copeia, 2005, 320-339.

Eger JL, Mitchell L (2003) Chiroptera, Bats. In: The Natural History of Madagascar (eds Goodman SM, Benstead JP), pp. 1287-1298. The University of Chicago Press, Chicago, Illinois.

Emerson BC (2002) Evolution on oceanic islands: molecular phylogenetic approaches to understanding pattern and process. Molecular Ecology, 11, 951-966.

Evans BJ, Brown RM, McGuire JA et al. (2003) Phylogenetics of fanged frogs: testing biogeographical hypotheses at the interface of the Asian and Australian faunal zones. Systematic Biology, 52, 794-819.

Excoffier L, Smouse PE, Quattro JM (1992) Analysis of molecular variance inferred from metric distances among DNA haplotypes: application to human mitochondrial DNA restriction data. Genetics, 131, 479-491.

Fu YX (1997) Statistical tests of neutrality of mutations against population growth, hitchhiking, and background selection. Genetics, 147, 915-925.

Fu YX, Li WH (1993) Statistical tests of neutrality of mutations. Genetics, 133, 693-709.

Garbutt N (1999) Mammals of Madagascar. Yale University Press, New Haven, Connecticut.

Givnish TJ, Millam KC, Evans TM et al. (2004) Ancient vicariance or recent long-distance dispersal? Inferences about phylogeny and South American-African disjunctions in Rapateaceae and Bromeliaceae based on $n d h \mathrm{~F}$ sequence data. International Journal of Plant Sciences, 165, S35-S54.

Goodman SM, Andriafidison D, Andrianaivoarivelo R et al. (2005) The distribution and conservation of bats in the dry regions of Madagascar. Animal Conservation, 8, 153-165.

von Haeseler A, Sajantila A, Pääbo S (1996) The genetical archaeology of the human genome. Nature Genetics, 14, 135-140.

Hastenrath S, Greischar L (1991) The monsoonal current regimes of the tropical Indian Ocean: observed surface flow fields and their geostrophic and wind-driven components. Journal of Geophysical Research, 96, 12619-12633.

Heads M (2005) Dating nodes on molecular phylogenies: a critique of molecular biogeography. Cladistics, 21, 62-78.
Hey J, Nielsen R (2004) Multilocus methods for estimating population sizes, migration rates and divergence time, with applications to the divergence of Drosophila pseudoobscura and D. persimilis. Genetics, 167, 747-760.

Hill JE (1982) A review of the leaf-nosed bats Rhinonycteris, Cloeotis and Triaenops (Chiroptera: Hipposideridae). Bonner Zoologische Beiträge, 33, 165-186.

Huelsenbeck JP, Ronquist F (2001) MRBAYEs: Bayesian inference of phylogenetic trees. Bioinformatics, 17, 754-755.

Hulva P, Horácek I, Strelkov PP, Benda P (2004) Molecular architecture of Pipistrellus pipistrellus/Pipistrellus pygmaeus complex (Chiroptera: Vespertilionidae): further cryptic species and Mediterranean origin of the divergence. Molecular Phylogenetics and Evolution, 32, 1023-1035.

King CM (1990) The Handbook of New Zealand Mammals. Oxford University Press, New York.

Kingston T, Lara MC, Jones G et al. (2001) Acoustic divergence in two cryptic Hipposideros species: a role for social selection? Proceedings of the Royal Society. Series B, Biological Sciences, 268, 1381-1386.

Kocher TD, Thomas WK, Meyer A et al. (1989) Dynamics of mitochondrial DNA evolution in animals: amplification and sequencing with conserved primers. Proceedings of the National Academy of Sciences, USA, 86, 6196-6200.

Kuhner MK (2004) LAMARC: estimating population genetic parameters from molecular data. In: The Phylogenetic Handbook: a Practical Approach to DNA and Protein Phylogeny (eds Salemi M, Vandamme A-M), pp. 378-399. Cambridge University Press, London.

Kuhner MK (2006) LAMARC 2.0: maximum likelihood and Bayesian estimation of population parameters. Bioinformatics, 22, 768770 .

Maddison DR, Maddison WP (2000) MACCLADE 4: Analysis of Phylogeny and Character Evolution, Version 4.0. Sinauer Associates, Sunderland, Massachusetts.

McCracken GF, Hayes JP, Cevallos J, Guffey SZ, Romero FC (1997) Observations on the distribution, ecology, and behaviour of bats on the Galapagos Islands. Journal of Zoology, 243, 757-770.

McDonald JH, Kreitman M (1991) Adaptive protein evolution at the Adh locus in Drosophila. Nature, 351, 652-654.

Near TJ, Keck BP (2005) Dispersal, vicariance, and timing of diversification in Nothonotus darters. Molecular Ecology, 14, 3485-3496.

Nelson G, Platnick NI (1981) Systematics and Biogeography: Cladistics and Vicariance. Columbia University Press, New York.

Peterson RL, Eger JL, Mitchell L (1995) Chiroptères. Muséum National d'Histoire Naturelle, Paris.

Posada D, Crandall KA (1998) MODELTEST: testing the model of DNA substitution. Bioinformatics, 14, 817-818.

Ramos-Onsins SE, Rozas J (2002) Statistical properties of new neutrality tests against population growth. Molecular Biology and Evolution, 19, 2092-2100.

Ranivo J, Goodman SM (in press) Révision taxinomique des Triaenops malgaches (Mammalia: Chiroptera: Hipposideridae). Zoosystema.

Rannala B, Yang Z (2003) Bayes estimation of species divergence times and ancestral population sizes using DNA sequences from multiple loci. Genetics, 164, 1645-1656.

Raxworthy CJ, Forstner MRJ, Nussbaum RA (2002) Chameleon radiation by oceanic dispersal. Nature, 415, 784-787.

Rozas J, Sánchez-DelBarrio JC, Messeguer X, Rozas R (2003) DNASP, DNA polymorphism analyses by the coalescent and other methods. Bioinformatics, 19, 2496-2497. 
Sakai T, Kikkawa Y, Tsuchiya K et al. (2003) Molecular phylogeny of Japanese Rhinolophidae based on variations in the complete sequence of the mitochondrial cytochrome $b$ gene. Genes and Genetic Systems, 78, 179-189.

Sawyer S (1977) Asymptotic properties of the equilibrium probability of identity in a geographically structured population. Advances in Applied Probability, 9, 268-282.

Schneider S, Roessli D, Excoffier L (2001) ARLEQUIN, Version 2.001: a Software for Population Genetics Data Analysis. Genetics and Biometry Laboratory, University of Geneva.

Simmons NB (2005) Order Chiroptera. In: Mammal Species of the World: a Taxonomic and Geographic Reference, 3rd edn (eds Wilson DE, Reeder DM), pp. 312-529. Smithsonian Institution Press, Washington, D.C.

Slatkin M (1991) Inbreeding coefficients and coalescence times. Genetical Research, 58, 167-175.

Stone CP, Pratt LW (1994) Hawaii's Plants and Animals: Biological Sketches of Hawaii Volcanoes National Park. University of Hawaii Press, Honolulu.

Storey M, Mahoney JJ, Saunders AD et al. (1995) Timing of hot spot-related volcanism and the breakup of Madagascar and India. Science, 267, 852-855.

Swofford DL (1998) PAUP*: Phylogenetic Analysis Using Parsimony (*and Other Methods), Version 4.0b10. Sinauer Associates, Sunderland, Massachusetts.

Teeling EC, Springer MS, Madsen O et al. (2005) A molecular phylogeny for bats illuminates biogeography and the fossil record. Science, 307, 580-584.

Wakeley J (1996) Distinguishing migration from isolation using the variance of pairwise differences. Theoretical Population Biology, 49, 369-386.

Wang H, Liang B, Feng J, Sheng L, Zhang S (2003) Molecular phylogenetics of hipposiderids (Chiroptera: Hipposideridae) and rhinolophids (Chiroptera: Rhinolophidae) in China based on mitochondrial cytochrome $b$ sequences. Folia Zoologica, 52, 259-268.
Wickström LM, Haukisalmi V, Varis S, Hantula J, Henttonen H (2005) Molecular phylogeny and systematics of anoplocephaline cestodes in rodents and lagomorphs. Systematic Parasitology, 62, 83-99.

Yang Z (2002) Likelihood and Bayes estimation of ancestral population sizes in hominoids using data from multiple loci. Genetics, 162, 1811-1823.

Yoder AD, Burns MM, Zehr S et al. (2003) Single origin of Malagasy Carnivora from an African ancestor. Nature, 421, 734-737.

Yoder AD, Olson LE, Hanley C et al. (2005) A multidimensional approach for detecting species patterns in Malagasy vertebrates. Proceedings of the National Academy of Sciences, USA, 102, 65876594.

Yoder AD, Yang Z (2004) Divergence dates for Malagasy lemurs estimated from multiple gene loci: geological and evolutionary context. Molecular Ecology, 13, 757-773.

This work was conducted as part of Amy Russell's postdoctoral fellowship at Yale University. Amy is currently a postdoctoral associate at the University of Arizona, and is interested in the impact of sex-specific behaviours on patterns of genetic diversity in natural populations. Julie Ranivo is a graduate student completing her thesis on the ecomorphology and taxonomy of Malagasy bats. Eric Palkovacs is a Ph.D. student interested in the applications of molecular genetic techniques to questions of ecology, biogeography and conservation. Steven Goodman is actively involved in documenting the current land vertebrate diversity of Madagascar and its conservation. Anne Yoder has long been interested in Madagascar as a natural laboratory for generating vertebrate diversity. She has used phylogenetic methods to reconstruct the evolutionary history of lemurs and other Malagasy vertebrates. 